\title{
Active noise control using a functional link artificial neural network with the simultaneous perturbation learning rule
}

\author{
Ya-li Zhou ${ }^{\mathrm{a}, *}$, Qi-zhi Zhang ${ }^{\mathrm{a}}$, Tao Zhang ${ }^{\mathrm{a}}$, Xiao-dong $\mathrm{Li}^{\mathrm{b}}$ and Woon-seng Gan ${ }^{\mathrm{c}}$ \\ ${ }^{a}$ Department of Computer Science and Automation, Beijing Institute of Machinery, P.O. Box 2865, Beijing, 100192, \\ P.R. China \\ ${ }^{\mathrm{b}}$ Institute of Acoustics, Academia Sinica, P.R. China \\ ${ }^{\mathrm{C}}$ School of EEE, Nanyang Technological University, Singapore
}

Received 13 July 2007

Revised 2008

\begin{abstract}
In practical active noise control (ANC) systems, the primary path and the secondary path may be nonlinear and timevarying. It has been reported that the linear techniques used to control such ANC systems exhibit degradation in performance. In addition, the actuators of an ANC system very often have nonminimum-phase response. A linear controller under such situations yields poor performance. A novel functional link artificial neural network (FLANN)-based simultaneous perturbation stochastic approximation (SPSA) algorithm, which functions as a nonlinear mode-free (MF) controller, is proposed in this paper. Computer simulations have been carried out to demonstrate that the proposed algorithm outperforms the standard filtered-x least mean square (FXLMS) algorithm, and performs better than the recently proposed filtered-s least mean square (FSLMS) algorithm when the secondary path is time-varying. This observation implies that the SPSA-based MF controller can eliminate the need of the modeling of the secondary path for the ANC system.
\end{abstract}

Keywords: Active noise control, FLANN, SPSA

\section{Introduction}

The exponential increase of noise pollution and ineffectiveness of passive techniques for noise attenuation have led to the development of active noise control (ANC) system [1-6]. This technique has been successfully applied to heating, ventilating, and air conditioning systems [4,5], exhaust and motor noise [4,7], headsets [4,6], and airplanes [4]. ANC, in general, is based on the principle of destructive interference between an original primary noise source and a secondary source, whose acoustic output is governed by a controller. A typical ANC system in a duct is shown in Fig. 1. The noise from the primary source travels as plane waves from left to right through the duct. The reference microphone that is located upstream from the secondary source detects the incident noise waves and supplies the controller with a reference signal $x(n)$. The controller sends a control signal $y(n)$ to the secondary source (i.e., loudspeaker), to attenuate the primary acoustic noise in the duct. The error microphone that is located downstream, picks up the residuals and supplies the controller with an error signal $e(n)$ [1].

The controller has to be adaptive because of changes in the environment, degradation of system components (e.g., loudspeaker), and alterations of the noise source [4]. Most of the conventional ANC systems are linear in nature and they employ the standard filtered-x least mean square (FXLMS) algorithm [1,2]. Even though this algorithm

\footnotetext{
*Corresponding author. E-mail: zhouyali@yahoo.com.
} 


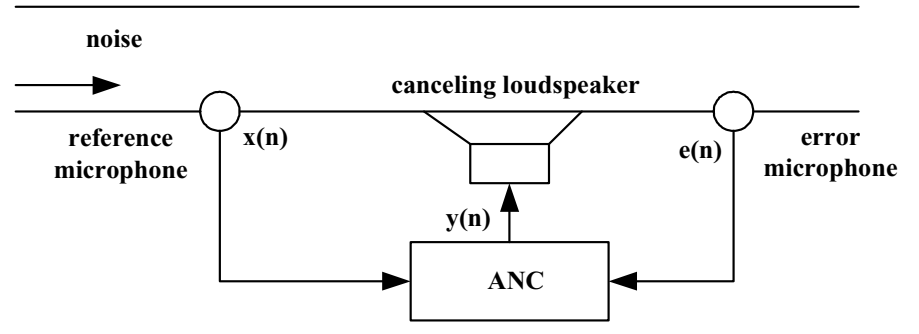

Fig. 1. Feedforward ANC system in a duct.

involves less computational complexity for active control of broadband and narrowband noise, it may not perform well in the cases where the ANC system exhibits nonlinear response characteristics [4].

Nonlinearities may be present in the primary path when the noise is propagating with high sound pressure [8] and the nonlinearity of the air is taken into account. Nonlinearities can also occur in the secondary path due to the use of A/D and D/A converters, power amplifiers, loudspeakers and transducers. Overdriving the electronics or the loudspeakers gives rise to relevant nonlinear effects [9].

To deal with these effects, different structures for nonlinear controllers have been recently proposed in the literature [3,4,7-17]. In [3], a nonlinear controller based on a filtered-error BP neural network was proposed to offer computational advantage over the filtered-x BP neural network algorithm. In [4], a nonlinear controller based on a normalized Gaussian radial basis neural network was proposed to compensate the nonminimum-phase secondary path transfer function and control the nonlinear noise process. In [10,11], radial basis function (RBF) neural networks were used to build intelligent controllers that remember the optimal control parameters corresponding to different linear plant configurations for the active control of sound. Neural networks and recurrent neural networks have also been used [12-14] to control the nonlinear effects in the secondary path transfer function. In [7], adaptive Volterra filters have been introduced for feedforward ANC.

In the recent literature [15-21], structures based on Functional Link Artificial Neural Network (FLANN) have attracted much research attention. The basic principle of FLANN is to expand the dimensionality of the input signal space by using a set of linearly independent functions [19]. Several mapping functions have been used to achieve this purpose, such as Legendre, Chebyshev, and trigonometric polynomials. Among these choices, the Fourier series being composed of the linear combination of trigonometric harmonics is generally recognized as one of the best approximating functions [20].

The major difference between the hardware structures of multi-layer perception (MLP) and FLANN is that FLANN has only input and output layers, and the hidden layers are completely replaced by the nonlinear mappings. In fact, the task performed by the hidden layers in an MLP is carried out by functional expansions in FLANN [21]. Since the input signals are nonlinearly mapped into the output signal space, the FLANN also has the ability to resolve the nonlinear problems. At the same time, the FLANN has a much simpler structure than a MLP, it has the advantage of involving lesser computational complexity and faster convergence rate than a MLP [16,18,19,21]. In two recent papers [16,17], a single-layer FLANN with a new filtered-s LMS (FSLMS) algorithm has been employed for nonlinear active noise control.

Observations of various structures and algorithms reported in [3,4,7-21] reveal that the structure based on FLANN with the FSLMS algorithm is a preferable nonlinear structure for active control of nonlinear noise processes because of its interesting properties. But, in this control method, in order to update the weights of the FLANN, we need a gradient of the error function, namely, we must know the model of the secondary path [3].

However, characteristics of the secondary path usually vary with respect to temperature or other environments, that is, the secondary path is time-varying. Therefore, it is difficult to estimate the exact characteristics of the secondary path accurately [22]. To solve this problem, a model-free (MF) control scheme based on the simultaneous perturbation stochastic approximation (SPSA) algorithm is proposed here [23]. This approach is based on the output error of the system to update the weights of the FLANN without the need to model the secondary path. It requires only two objective function measurements regardless of the number of weights being updated, it uses simultaneous perturbation (SP) to update all weights of the FLANN simultaneously [24-28]. 


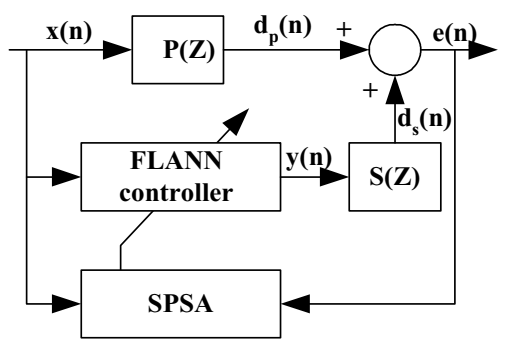

Fig. 2. The block diagram of an ANC system.

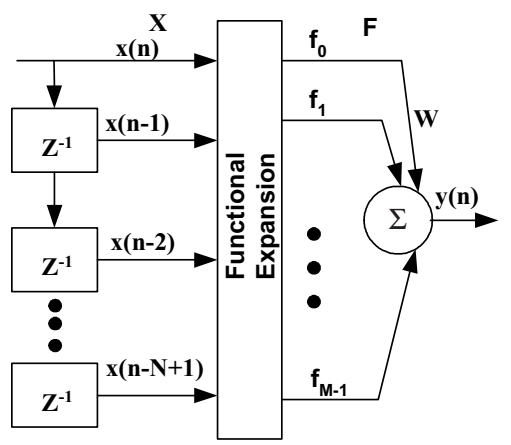

Fig. 3. The FLANN controller.

The organization of the paper is as follows. In Section 2, a new FLANN-based SPSA algorithm is developed in this Section. The simulation results using the proposed algorithm and other conventional algorithms are shown in Section 3. Finally, conclusions are presented in Section 4.

\section{Control algorithm}

The block diagram of a feedforward ANC system using the FLANN-based SPSA algorithm is shown in Fig. 2. The primary-path $P(Z)$ is from the noise source to the error microphone, and the secondary path $S(Z)$ is from the canceling loudspeaker to the error microphone. The FLANN controller is used to generate the control signal $y(n)$. Note that this algorithm does not use the estimator of the secondary path [28].

Figure 3 shows the structure of the FLANN controller. At any time instant $n$, defining the input signal vector $\boldsymbol{X}(n)$ as

$$
\boldsymbol{X}(n)=[x(n), x(n-1), \cdots x(n-N+1)]^{T} .
$$

Where $N$ is the length of the input vector $\boldsymbol{X}(n),[.]^{T}$ denotes transpose of a vector.

Then the functionally expanded vector $\boldsymbol{F}(n)$ is $[16,18,19]$

$$
F(n)=\left[f_{0}(n), f_{1}(n), \cdots f_{M-1}(n)\right]^{T}=[F E(x(n)), F E(x(n-1)), \cdots F E(x(n-N+1))]^{T} .
$$

Where $F E($.$) stands for functional expansion, M$ is the length of the functional enhanced vector $\boldsymbol{F}(n)$.

These $M$ linearly independent functions map the $N$-dimensional signal space into an $M$-dimensional space, that is $\boldsymbol{R}^{N} \rightarrow \boldsymbol{R}^{M}, N<M$.

$\boldsymbol{W}(n)$ is the weight vector and is given by

$$
\boldsymbol{W}(n)=\left[w_{0}(n), w_{1}(n), \cdots w_{M-1}(n)\right]^{T} .
$$

It is common knowledge that if the secondary path of the ANC system is completely unknown, it is impossible to use usual gradient method as a learning rule to update the controller coefficients $[25,27,28]$. In this case, an estimator 
of the gradient of the error function is needed. The SPSA which was introduced by J.C. Spall [23] is a well-known gradient approximation approach that relies on measurements of the objective function, not on measurements of the gradient of the objective function.

The objective of the following analysis is to develop the FLANN-based SPSA algorithm to improve the noise cancellation capability of a nonlinear ANC system.

\section{Step 1: Define the error function}

Note that in ANC system, each sampling error signal does not contain enough information as an evaluation function to be optimized. That is, the expectation of the error signal has to be used as the evaluation function. For practicality, the sum of the error signal for a certain interval is used to approximate the expectation of the error signal. Thus, the error function is defined as [22]

$$
J(y(n))=\frac{1}{2} \sum_{n=1}^{\lambda} e^{2}(n)=\frac{1}{2} \sum_{n=1}^{\lambda}\left[d_{s}(n)+d_{p}(n)\right]^{2} .
$$

where $n$ is the sampling number in a block interval, and $\lambda$ is the total sampling number of one block interval.

Step 2: Functional expansion of the input pattern vector $\boldsymbol{X}(n)$

For functional expansion of the input pattern, we have chosen the trigonometric polynomials. Then the functionally expanded vector $\boldsymbol{F}(n)$ can be written as $[16,17]$

$$
\begin{aligned}
F(n)= & {\left[f_{0}(n), f_{1}(n), \cdots f_{M-1}(n)\right]^{T}=[x(n), \sin [\pi x(n)], \cos [\pi x(n)], \cdots \sin [P \pi x(n)], \cos [P \pi x(n)] \cdots} \\
& x(n-1), \sin [\pi x(n-1)], \cos [\pi x(n-1)], \cdots \sin [P \pi x(n-1)], \cos [P \pi x(n-1)] \cdots \\
& x(n-N+1), \sin [\pi x(n-N+1)], \cos [\pi x(n-N+1)], \cdots \sin [P \pi x(n-N+1)], \\
& \cos [P \pi x(n-N+1)]]^{T} .
\end{aligned}
$$

Where $P$ is the order of functional expansion, then $M=N(2 P+1)$.

Step 3: Compute the anti-noise signal $d_{s}(n)$

The output of the FLANN is

$$
y(n)=W^{T}(n) F(n)=\sum_{j=0}^{M-1}\left[w_{j}(n) f_{j}(n)\right] .
$$

Then the anti-noise signal $d_{s}(n)$ can be calculated using the following equation

$$
d_{s}(n)=S(n)^{T} Y(n)=\sum_{j=0}^{L-1} s_{j}(n) y(n-j) .
$$

Where $\boldsymbol{S}(n)=\left[s_{0}(n) s_{1}(n) \ldots s_{L-1}(n)\right]^{T}$, is the impulse response of the secondary path transfer function $S(Z), L$ is the length of the secondary path. $\boldsymbol{Y}(n)=[y(n) y(n-1) \ldots y(n-L+1)]^{T}$.

\section{Step 4: Generation of SP vector}

The following perturbation vector $\Delta(n)$ is generated as independent Bernoulli random variables with outcomes of \pm 1 that gives small disturbances to all weights [26,28]

$$
\Delta(n)=\left(\Delta_{0}(n), \Delta_{1}(n), \cdots \Delta_{M-1}(n)\right)^{T} .
$$

\section{Step 5: Error function evaluations}

Obtain two measurements of the error function $J(\cdot)$ based on the SP: $J\left(y\left(W(n)+c_{k} \Delta(n)\right)\right)$ and $J(y(W(n)-$ $\left.\left.c_{k} \Delta(n)\right)\right)$ with the $\Delta(n)$ from step 4 .

Where $c_{k}$ is a positive scalar and represents a magnitude of the perturbation. 


\section{Step 6: Gradient approximation}

$$
\begin{aligned}
& \text { Generate the SP approximation to the unknown gradient } \frac{\partial J(y(\boldsymbol{W}(n)))}{\partial \boldsymbol{W}(n)} \text { as [26-28] } \\
& \Delta \boldsymbol{W}(n)=\frac{J\left(y\left(\boldsymbol{W}(n)+c_{k} \Delta(n)\right)\right)-J\left(y\left(\boldsymbol{W}(n)-c_{k} \Delta(n)\right)\right)}{c_{k} \Delta(n)} .
\end{aligned}
$$

Step 7: Update the weight vector $\boldsymbol{W}(n)$ of the FLANN

Weights of the FLANN are updated in the following manner:

$$
\boldsymbol{W}(n+1)=\boldsymbol{W}(n)-a_{k} \Delta \boldsymbol{W}(n) .
$$

Where $a_{k}$ is a positive learning coefficient.

From Eqs (9) and (10), it can be seen that the weights of the FLANN controller is updated without the need to model the secondary path, so this algorithm is called MF control algorithm.

\section{Simulation studies}

To demonstrate the effectiveness of the proposed algorithm, computer simulations are performed on various nonlinear situations in an ANC system. In these simulations, both minimum-phase and nonminimum-phase transfer functions for the secondary path are considered. At the same time, a comparison between the proposed algorithm, the FSLMS algorithm and the FXLMS algorithm is made.

Case 1: A simple linear ANC example is first considered to illustrate the FLANN-based SPSA algorithm effectiveness. For the purpose of comparison, both FXLMS algorithm and FSLMS algorithm are also simulated. The reference signal $x(n)$ is the sum of a sinusoidal wave of $300 \mathrm{~Hz}$ sampled at the rate of $3000 \mathrm{samples} / \mathrm{s}$ and a Gaussian white noise.

The primary path transfer function is considered to be [3]

$$
P(Z)=0.8 Z^{-6}+0.6 Z^{-7}-0.2 Z^{-8}-0.5 Z^{-9}-0.1 Z^{-10}+0.4 Z^{-11}-0.05 Z^{-12} \text {. }
$$

The secondary path transfer function is taken as the minimum-phase model and is assumed to be time-invariant

$$
\begin{aligned}
S(Z)= & 0.3 Z^{-2}+0.4 Z^{-3}+0.1 Z^{-4}-0.2 Z^{-5}-0.1 Z^{-6}+0.1 Z^{-7}+0.1 Z^{-8} \\
& +0.01 Z^{-9}+0.001 Z^{-10} .
\end{aligned}
$$

For the FLANN-based SPSA algorithm and the FSLMS algorithm, the length of the input vector $\boldsymbol{X}(n)$ is $13(N=13)$, the functional expansion of the input signal is of first-order type $(P=1)$. For the FLANN-based SPSA algorithm, the total sampling number of one block interval $\lambda$ is set as $100 . c_{k}$ and $a_{k}$ are set as 0.001 and 0.0001 , respectively. the convergence coefficient for the FSLMS algorithm is set at $\mu=0.03$. For the FXLMS algorithm, a 13-tap finite impulse response (FIR) filter is used, the convergence coefficient for the FXLMS algorithm is set at $\mu=$ 0.001. The adaptation continues for 30000 iterations. The simulation result of the canceling error in the frequency domain is shown in Fig. 4. From the simulation result shown in Fig. 4, it may be seen that when the secondary path model is deterministic and time-invariant, the noise-canceling performance of the FLANN-based SPSA algorithm is inferior compared to the FXLMS algorithm and the FSLMS algorithm. The reason is that the FLANN-based SPSA algorithm is a stochastic gradient approximation algorithm, it is based on using the SP function to approximate the gradient of the error function without the need to identify the system model [23-26]. It is generally inappropriate for systems where a reliable system model can be determined. One reason, of course, is that with a reliable model, the gradient of the error function can be calculated accurately, and thus the controller will generally achieve optimal control [24]. Although the FLANN-based SPSA algorithm has the aforementioned disadvantage, from Fig. 4, it may be seen that the noise-canceling performance of the FLANN-based SPSA algorithm is quite satisfactory, the major disturbance frequency is attenuated by approximately $60 \mathrm{~dB}$.

Case 2: Next, we deal with a tracking problem. For the purpose of comparison, the FSLMS algorithm is also simulated. With the same set of parameters used for case 1, when the number of iterations reaches 25,000, the 


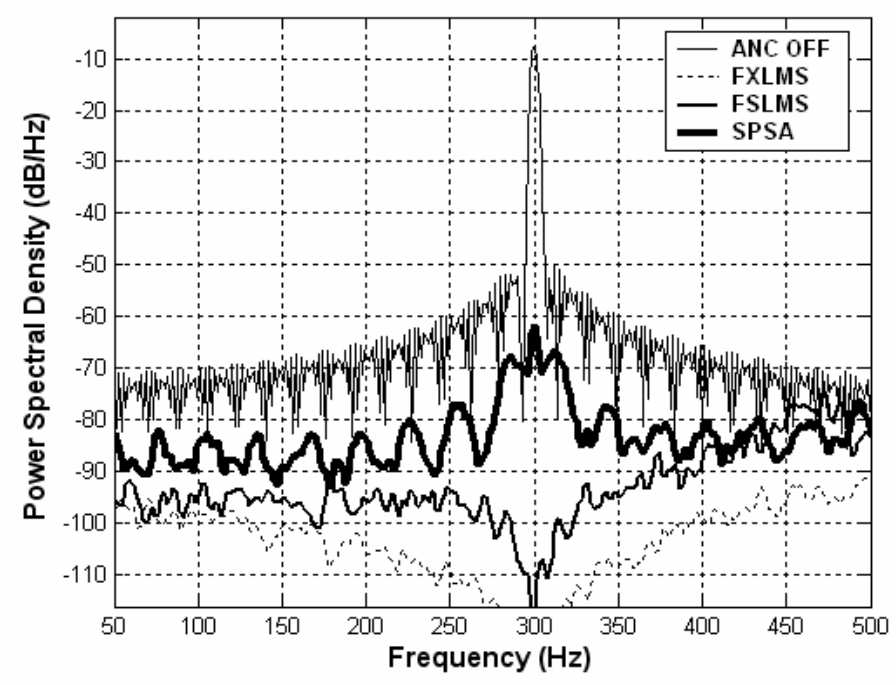

Fig. 4. The error signal spectrum for case 1.

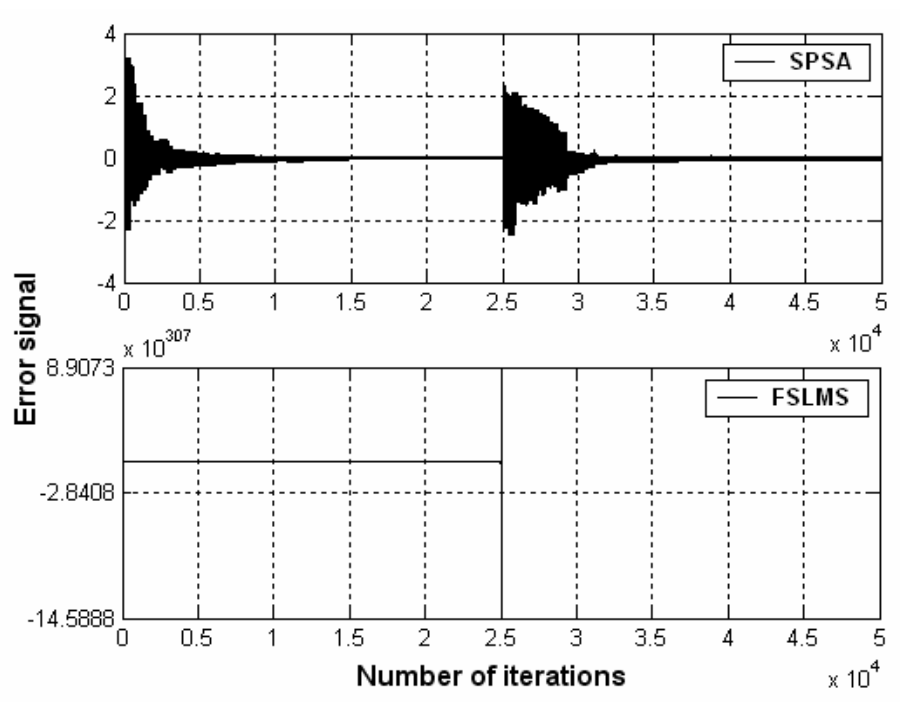

Fig. 5. The error signal versus number of iterations for case 2 .

secondary path is altered by letting $S(z)=-S(z)$. The adaptation continues for 50000 iterations. The error signal in error microphone versus the number of iterations is shown in Fig. 5. From the simulation result shown in Fig. 5, it may be seen that when the secondary path model is time-varying, the FLANN-based SPSA algorithm has a good tracking ability of the secondary path. After a short transient phase, the system settles down to a steady-state response. Whereas the FSLMS algorithm cannot adapt itself to the change of the secondary path because of its model-based characteristics [16]. From the simulation result shown in Fig. 5, it is concluded that the SPSA-based MF controller can eliminate the need of the modeling of the secondary path for the ANC system. So, such an approach has potential advantages in accommodating systems where the equations governing the system are unknown or with time-varying dynamics [24].

Case 3: In this case, the example of a nonlinear primary path is used in the simulation. For the purpose of 


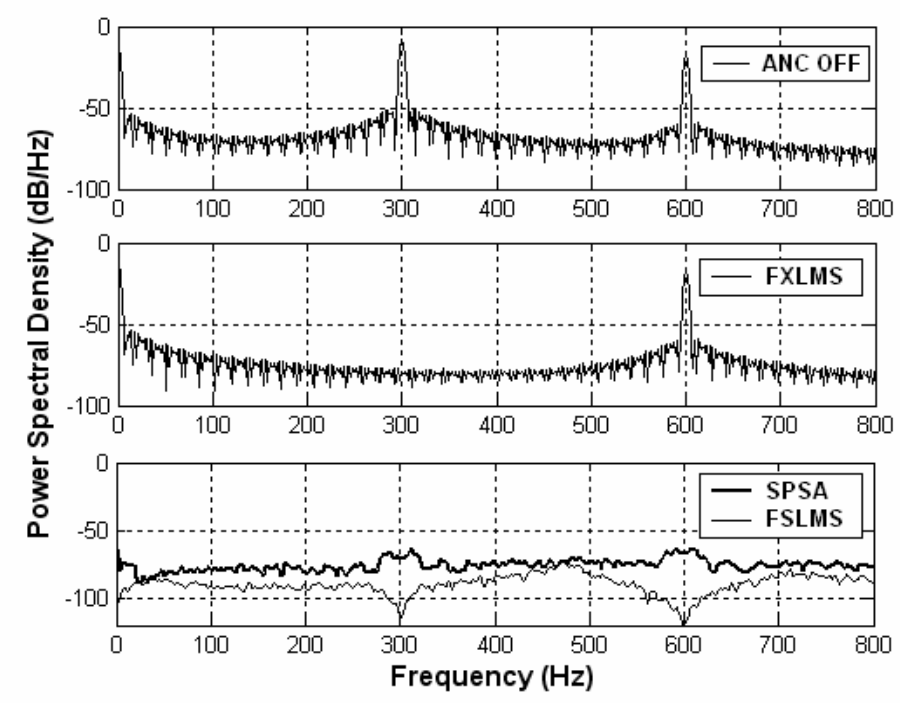

Fig. 6. The error signal spectrum for case 3 .

comparison, both the FXLMS algorithm and the FSLMS algorithm are also simulated. The secondary path transfer function is chosen to be the minimum-phase model of case 1 . The primary noise at the cancellation point $d_{p}(n)$ is generated based on the following second-order polynomial model

$$
d_{p}(n)=u(n-2)+0.6 u^{2}(n-2) .
$$

Where

$$
u(n)=\boldsymbol{P}^{T}(n) \boldsymbol{X}(n)=\sum_{j=0}^{H-1}\left[p_{j}(n) x(n-j)\right] .
$$

Where $\boldsymbol{P}(n)=\left[p_{0}(n) p_{1}(n) \ldots p_{H-1}(n)\right]^{T}$, is the impulse response of the primary-path transfer function $P(Z), H$ is the length of the primary path.

Other parameters are same as those of case 1. Figure 6 presents the simulation result of the canceling error in the frequency domain. From this plot, it is evident that all the three algorithms can reduce the $300 \mathrm{~Hz}$ sinusoidal signal effectively. But only the FSLMS and the FLANN-based SPSA algorithm are effective on the harmonic noise signal caused by nonlinearity. Figure 7 shows the error signal in error microphone versus the number of iterations. When the number of iterations reaches 25,000 , the secondary path is altered by letting $S(z)=-S(z)$. From the result shown in Fig. 7, it may be seen that for the nonlinear primary path, the FLANN-based SPSA algorithm also has a good tracking ability of the secondary path.

Case 4: In this case, the examples of a nonlinear secondary path are used in the simulation. Two kinds of nonlinearities are considered, namely, NL1 and NL2. The nonlinear model NL1 corresponds to systems suffering from nonlinear distortion which is possibly caused by the saturation of amplifiers used in the secondary path. The nonlinear model NL2 is simply arbitrary nonlinear function [20]. For the model NL1, The anti-noise signal at the cancellation point $d_{s}(n)$ is generated based on the following expression

$$
d_{s}(n)=\tanh (u(n)) \text {. }
$$

Where

$$
u(n)=\boldsymbol{S}^{T}(n) \boldsymbol{Y}(n)=\sum_{j=0}^{L-1} s_{j}(n) y(n-j) .
$$

For the model NL2, the anti-noise signal at the cancellation point $d_{s}(n)$ is generated based on the following third-order polynomial model 


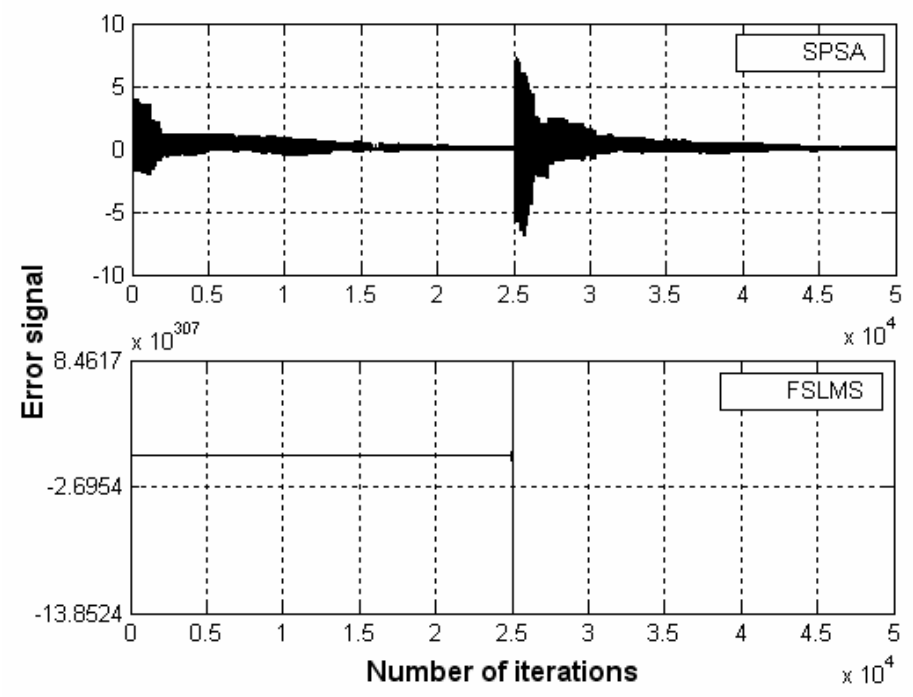

Fig. 7. The error signal versus number of iterations for case 3.

$$
d_{s}(n)=u(n)-0.1 u^{2}(n)+0.2 u^{3}(n) .
$$

The primary path is chosen to be the linear model of case 1 , the reference signal $x(n)$ is a sinusoidal wave of $300 \mathrm{~Hz}$ sampled at the rate of 6000 samples/s. For the model NL1, $a_{k}$ is set as 0.0003 , and for the model NL2, $a_{k}$ is set as 0.0005 . Other parameters are same as those of case 1. Figure 8 presents the simulation result of the canceling error in the frequency domain. From this plot, it is evident that the FLANN-based SPSA algorithm performs well in the case where the secondary path is nonlinear in an ANC system, the major disturbance frequency is attenuated by approximately $40 \mathrm{~dB}$.

Case 5: In this case, the reference signal used is same as this of case 1, both the linear primary path of case 1 and the nonlinear primary path of case 3 are taken for simulation study. The secondary path transfer function is chosen to be the nonminimum-phase model [16]

$$
S(Z)=Z^{-2}+1.5 Z^{-3}-Z^{-4} \text {. }
$$

Other parameters are same as those of case 1. Figure 9 presents the simulation result of the canceling error in the frequency domain. From Fig. 9, it may be seen that the FLANN-based SPSA algorithm performs well in the case where the secondary path transfer function is a nonminimum-phase model, the major disturbance frequency is attenuated by approximately $50 \mathrm{~dB}$ for both the linear primary path and the nonlinear primary path. For the nonlinear primary path, the harmonic noise signal caused by nonlinearity is attenuated by approximately $40 \mathrm{~dB}$.

\section{Conclusions}

In this paper, a novel FLANN-based SPSA algorithm is developed for use in a feedforward ANC system for situations where the primary path and the secondary path may be nonlinear and time-varying. The proposed algorithm is derived using the FLANN as the basic structure. This approach optimizes error function without using derivative of the error function. Therefore, the presented ANC algorithm does not require any estimation of the secondary path. Computer simulations have been carried out to assess the performance of the proposed algorithm as a candidate for nonlinear ANC. Its performance in terms of error power spectrum has been compared to that of standard FXLMS and the recently proposed FSLMS algorithm. It is shown that for the nonlinear control, in terms of the error power spectrum, the proposed algorithm outperforms the standard FXLMS algorithm. And it exhibits better performance than the FSLMS algorithm when the secondary path is time-varying. 


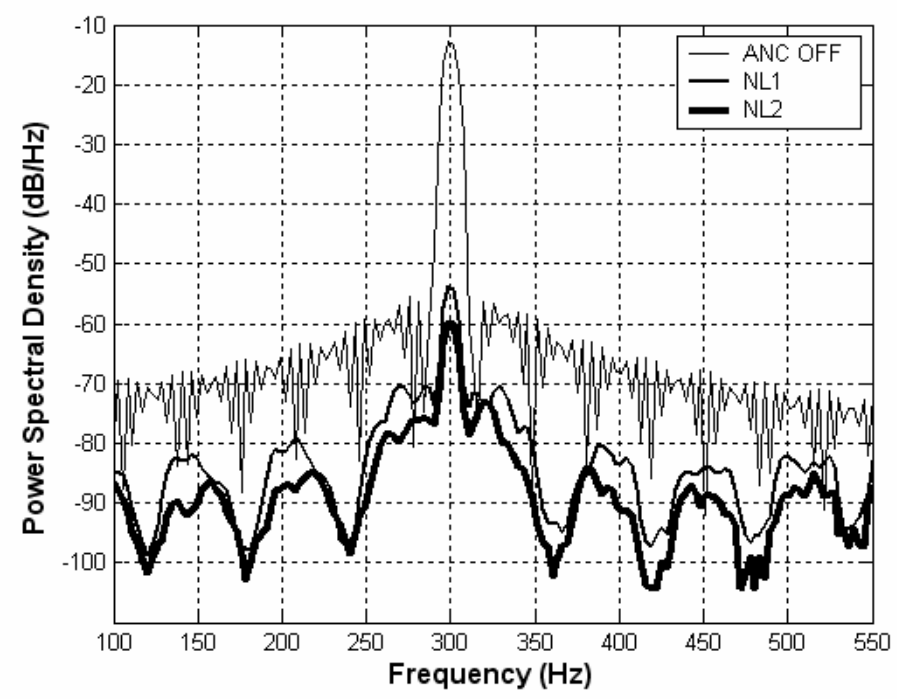

Fig. 8. The error signal spectrum for case 4.

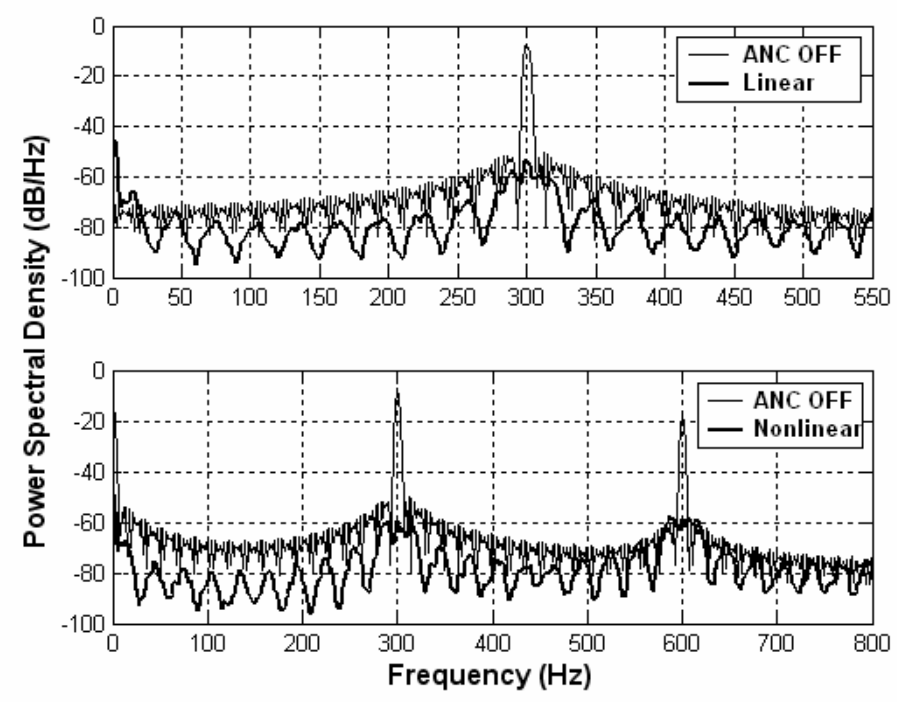

Fig. 9. The error signal spectrum for case 5.

\section{Acknowledgments}

This research is supported by Training Funds for Elitist of Beijing (20061D0500600164,20051A0500603).and Enhanced Teaching Plan Funds for Elitist in Colleges and Universities of Beijing .

\section{References}

[1] S.M. Kuo and D.R. Morgan, Active Noise Control Systems - Algorithms and DSP Implementations, Wiley, 1996.

[2] P.A. Nelson and S.J. Elliott, Active Sound Control, Academic Press, 1991. 
[3] Y.L. Zhou, Q.Z. Zhang, X.D. Li and W.S. Gan, Analysis and DSP Implementation of an ANC System using a Filtered-Error Neural Network, Journal of Sound and Vibration 285 (2005), 1-25.

[4] P. Strauch and B. Mulgrew, Active Control of Nonlinear Noise Processes in a Linear Duct, IEEE Trans Signal Processing 46 (1998), 2404-2412.

[5] H. Pelton, K.S. Wise and W.S. Sims, Active HVAC Noise Control System Provide Acoustical Comfort, Sound Vibration 28 (1994), 14-18.

[6] A.J. Salloway and C.E. Millar, Active Vibration and Noise Control, GEC Rev 11 (1996), 138-145.

[7] L. Tan, and J. Jiang, Adaptive Volterra Filter for Active Control of Nonlinear Noise processes, IEEE Trans Signal Processing 49 (2001), 1667-1676.

[8] W. Klippel, Active Attenuation of Nonlinear Sound, Patent, 1999.

[9] G.L. Sicuranza and A. Carini, On the Accuracy of Generalized Hammerstein Models for Nonlinear Active Noise Control, IMTC-2006, 2006, 1411-1416.

[10] M.O. Tokhi and R. Wood, Active Noise Control Using Radial Basis Function Networks, Contr Eng Practice 5 (1997), 1311-1322.

[11] T. Riyanto, L. Anggono and K. Uchida, DSP Based RBF Neural Modeling and Control for Active Noise Cancellation, Intelligent Control and Vancouver (2002), 460-466.

[12] M. Bouchard, B. Paillard and C.T.L. Dinh, Improved Training of Neural Networks for The Nonlinear Active Noise Control of Sound and Vibration, IEEE Trans Neural Networks 10 (1999), 391-401.

[13] S.D. Snyder and N. Tanaka, Active Control of Vibration using a Neural Network, IEEE Trans on Neural Networks 6 (1995), 819-828.

[14] B. Jayawardhana, L.H. Xie and S.Q. Yuan, Active Control of Sound based on Diagonal Recurrent Neural Network, SICE 2002, 2002, 2666-2671.

[15] D.P. Das, G. Panda and S. Sabat, Development of FLANN Based Multireference Active Noise Controllers for Nonlinear Acoustic Noise Processes, LNCS 3316, 2004, 1198-1203.

[16] D.P. Das and G. Panda, Active Mitigation of Nonlinear Noise Processes Using a Novel Filtered-s LMS Algorithm, IEEE Trans Speech Audio Processing 12 (2004), 313-322.

[17] D.P. Das, S.R. Mohapatra, A. Routray and T.K. Basu, Filtered-s LMS algorithm for multichannel active control of nonlinear noise processes, IEEE Transactions on Audio, Speech and Language Processing 14 (2006), 1875-1880.

[18] J.C. Patra, R.N. Pal, B.N. Chatterji and G. Panda, Identification of Nonlinear Dynamic System Using Functional Link Artificial Neural Networks, IEEE Trans Syst, Man, Cybernetics 29 (1999), 254-262.

[19] J.C. Patra and A.C. Kot, Nonlinear Dynamic System Identification Using Chebyshev Functional Link Artificial Neural Networks, IEEE Trans Syst, Man, Cybernetics 32 (2002), 505-511.

[20] W.D. Weng and C.T. Yen, Reduced-Decision Feedback FLANN Nonlinear Channel Equaliser for Digital Communication Systems, IEE Proc Сотти 151 (2004), 305-311.

[21] J.W. Zhang, K.Q. Wang and Q. Yue, Data Fusion Algorithm based on Functional Link Artificial Neural Networks, The 6th World Congress on Intelligent Control and Automation, 2006, 2806-2810.

[22] Y. Maeda and T. Yoshida, An Active Noise Control without Estimation of Secondary-Path, ACTIVE1999, 1999, 985-994.

[23] J.C. Spall, Multivariate Stochastic Approximation using Simultaneous Perturbation Gradient Approximation, IEEE Transactions on Automatic Control 37 (1992), 332-341.

[24] J.C. Spall and J.A. Cristin, Model-free control of nonlinear stochastic systems with discrete-time measurement, IEEE Transactions on Automatic Control 43 (1998), 1198-1210.

[25] J.C. Spall and J.A. Cristion, A Neural Network Controller for Systems with Unmodeled Dynamics with Applications to Wastewater Treatment, IEEE Transactions On Systems Man and Cybernetics 27 (1997), 369-375.

[26] J.C. Spall, Implementation of the Simultaneous Perturbation Algorithm for Stochastic Optimization, IEEE Transactions on Aerospace and Electronic Systems 34 (1998), 817-823.

[27] Y. Maeda and R.J.P. De Figueiredo, Learning Rules for Neuro-Controller via Simultaneous Perturbation, IEEE Transactions on Neural Networks 8 (1997), 1119-1130.

[28] Y.L. Zhou, Q.Z. Zhang, X.D. Li and W.S. Gan, Model-Free Control of a Nonlinear ANC System with a SPSA-based Neural Network Controller, Lecture Notes in Computer Science 3972 (2006), 1033-1038. 

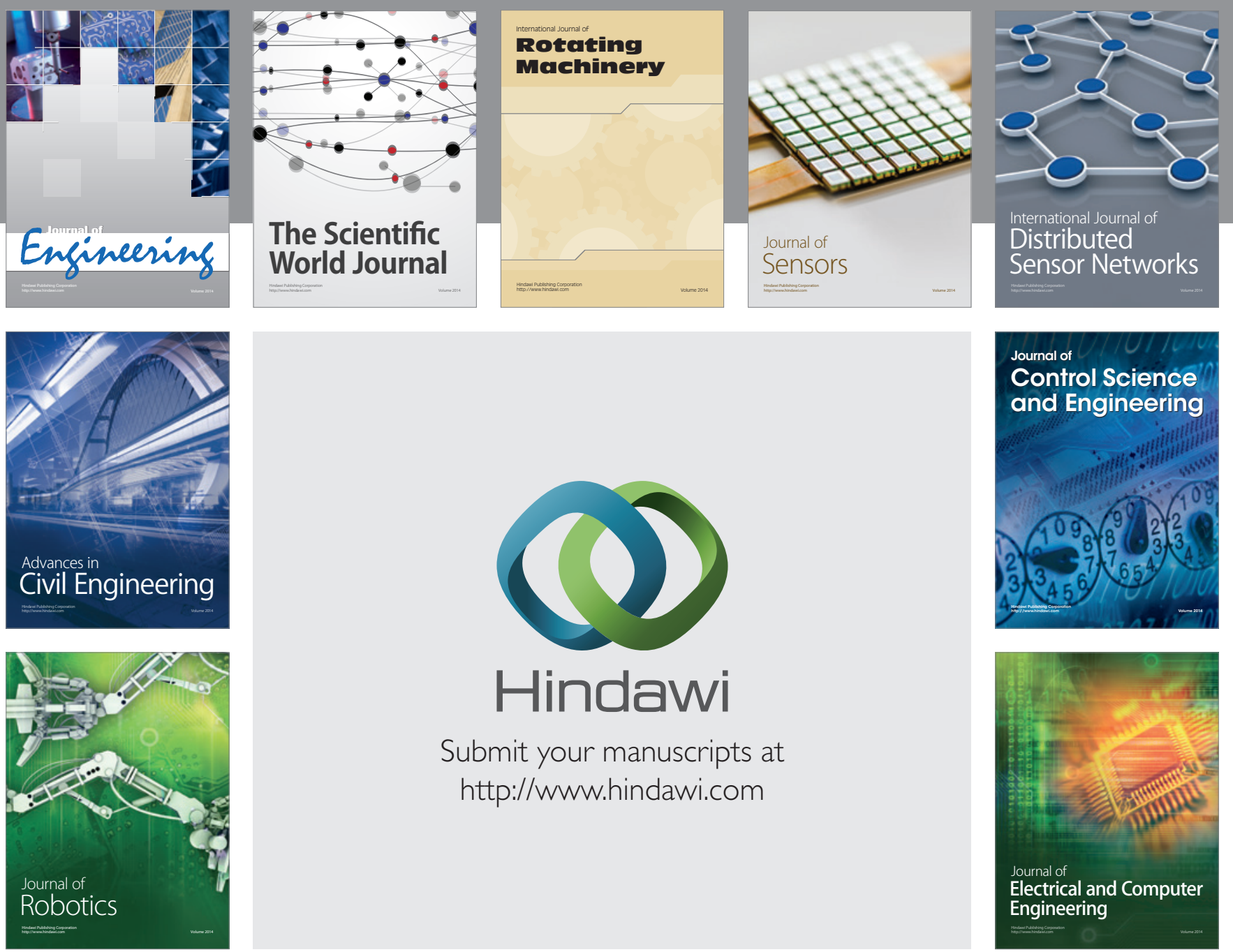

Submit your manuscripts at

http://www.hindawi.com
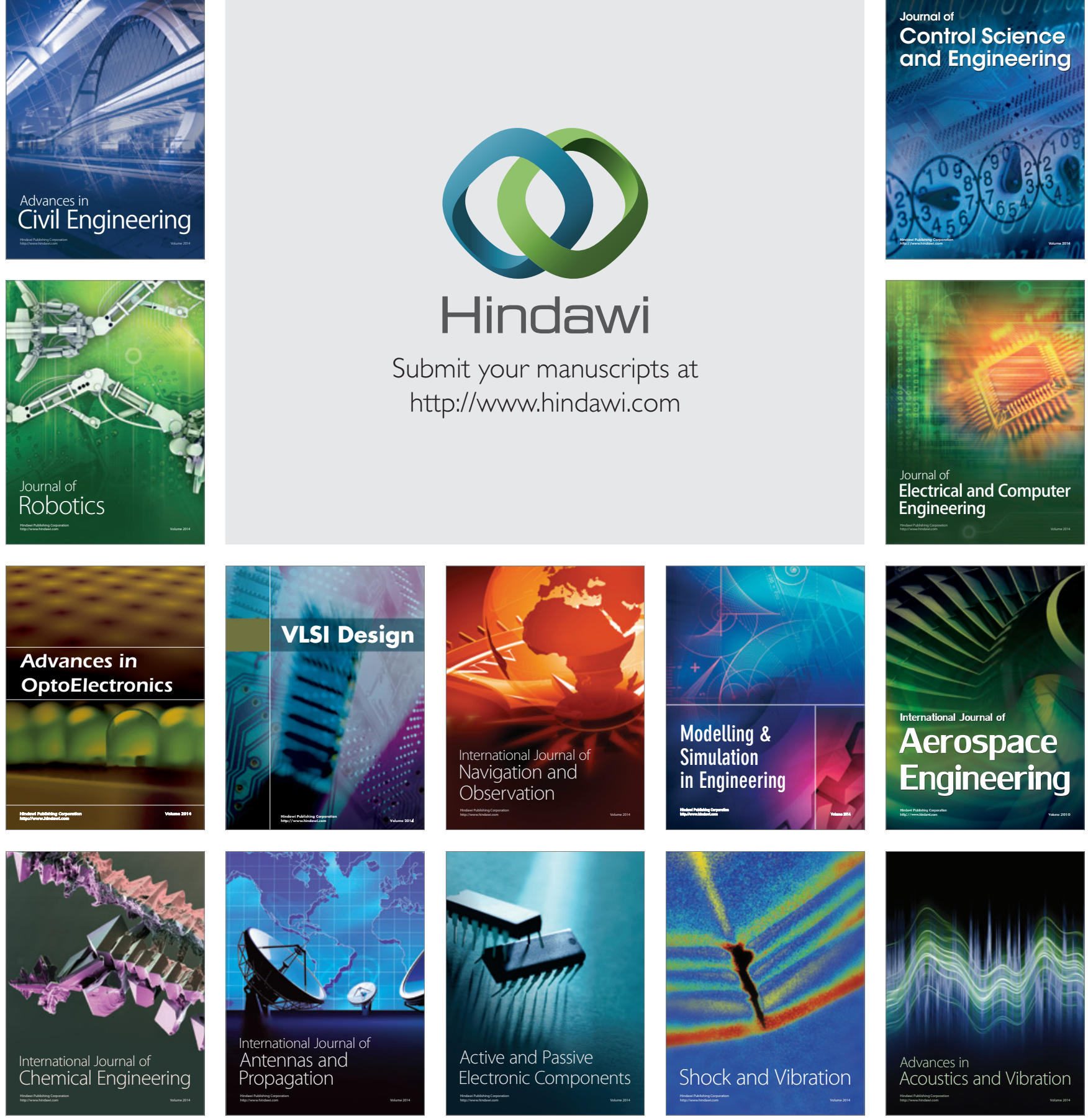